\title{
Sporadic Hypertrophic Cardiomyopathy Due to De Novo Myosin Mutations
}

Hugh Watkins, ** Ludwig Thierfelder, * Dar-San Hwang, ${ }^{*}$ William McKenna, ${ }^{*}$ J. G. Seidman,"' and C. E. Seidman * ${ }^{*}$ Cardiology Division, Brigham and Women's Hospital, and Harvard Medical School, Boston, Massachusetts 02115; ${ }^{\ddagger}$ Department of Cardiological Sciences, St. George's Hospital Medical School, London SW17 ORE, United Kingdom; ${ }^{8}$ Division of Cardiology, Taichung Veterans General Hospital, Taichung, Taiwan; "Department of Genetics, Harvard Medical School, Boston, Massachusetts 02115; and 'Howard Hughes Medical Inst., Boston, Massachusetts 02115

\begin{abstract}
Hypertrophic cardiomyopathy occurs as an autosomal dominant familial disorder or as a sporadic disease without familial involvement. While missense mutations in the $\beta$ cardiac myosin heavy chain (MHC) gene account for approximately half of all cases of familial hypertrophic cardiomyopathy, the molecular causes of sporadic hypertrophic cardiomyopathy are unknown. To determine whether $\beta$ cardiac MHC mutations are also associated with sporadic disease, we screened this gene in seven individuals with sporadic hypertrophic cardiomyopathy. Mutations in the $\beta$ cardiac MHC genes were identified in two probands with sporadic disease. In that their parents were neither clinically nor genetically affected, we conclude that mutations in each proband arose de novo. Transmission of the mutation and disease to an offspring occurred in one pedigree, predicting that these are germline mutations. The demonstration of hypertrophic cardiomyopathy arising within a pedigree coincident with the appearance of a de novo mutation provides compelling genetic evidence that $\beta$ cardiac MHC mutations cause this disease. We suggest that de novo mutations account for some instances of sporadic hypertrophic cardiomyopathy and that these mutations can be transmitted to children. The clinical benefits of defining mutations responsible for familial hypertrophic cardiomyopathy should also be available to some patients with sporadic disease. (J. Clin. Invest. 1992. 90:16661671.) Key words: cardiac hypertrophy $\bullet$ myosin heavy chain gene $\cdot$ RNase protection
\end{abstract}

\section{Introduction}

Hypertrophic cardiomyopathy is defined as a primary myocardial disorder $(1,2)$, characterized by increased ventricular mass with myocyte and myofibrillar disarray $(3,4)$, that occurs in the absence of other cardiac or systemic disorders. The diagnosis of hypertrophic cardiomyopathy is based upon the demonstration of unexplained ventricular hypertrophy. Hypertrophy is typically asymmetric, involving the septum and left ventricular free wall (5-7), but may be concentric or confined to the distal left ventricle and/or right ventricle (8-10). The familial form of hypertrophic cardiomyopathy is inherited as

Address reprint requests to Dr. Seidman, Department of Genetics, Harvard Medical School, 25 Shattuck Street, Boston, MA 02115. Received for publication 24 March 1992 and in revised form 10 June 1992.

J. Clin. Invest.

(C) The American Society for Clinical Investigation, Inc. 0021-9738/92/11/1666/06 \$2.00

Volume 90, November 1992, 1666-1671 an autosomal dominant trait $(5,11-14)$. Thus, individuals with familial hypertrophic cardiomyopathy frequently have multiple affected relatives, and must have one affected parent. Sporadic occurrence of hypertrophic cardiomyopathy is defined by similar anatomical and clinical features in an individual whose parents do not have the condition $(6,15)$.

Estimation of the fraction of hypertrophic cardiomyopathy that occurs as sporadic disease has been difficult. Two studies using two-dimensional echocardiography in families of probands with at least three available first degree relatives suggested that hypertrophic cardiomyopathy was sporadic in $44 \%$ (16) and $33 \%$ (17). However, clinical studies tend to overestimate the proportion of sporadic disease for several reasons. First, the severity and distribution of hypertrophy vary considerably, even between related family members, hence assignment of disease status may be inaccurate. Second, there are difficulties of ascertainment in retrospective studies where family members, especially parents, may be deceased or unavailable for study. Third, many of the families studied are too small for familial disease to manifest. For these reasons affected relatives may not be identified, with the result that the disease is assumed to be sporadic.

We have previously identified $\beta$ cardiac myosin heavy chain (MHC) ${ }^{1}$ mutations that cause familial hypertrophic cardiomyopathy in about $50 \%$ of unrelated families with this disorder (18-21). Seven different mutations have been identified; all are missense mutations affecting conserved residues in the amino-terminal half of the myosin heavy chain. Knowledge of the particular $\beta$ cardiac MHC gene mutation in a family with hypertrophic cardiomyopathy is of clinical importance, in terms of both diagnosis (20) and prognosis (21).

To investigate the molecular basis of sporadic hypertrophic cardiomyopathy we screened the $\beta$ cardiac MHC genes of seven unrelated probands with sporadic hypertrophic cardiomyopathy. Diagnosis was based on the presence of typical clinical features of the disease in the proband and the absence of disease criteria in both parents. We demonstrate that two of these probands have $\beta$ cardiac MHC mutations that are comparable to those found in patients with familial hypertrophic cardiomyopathy. Genetic analyses of the probands' parents reveal that both mutations have arisen de novo, coincident with the appearance of hypertrophic cardiomyopathy.

\section{Methods}

Clinical evaluation. After informed consent was obtained in accordance with the Brigham and Women's Hospital Committee for the

1. Abbreviations used in this paper: MHC, myosin heavy chain; STR, short tandem repeat (sequence). 
Protection of Human Subjects from Research Risks, blood was drawn from probands and their first-degree relatives. Clinical, electrocardiographic, and echocardiographic assessments were performed as described previously (22). The diagnosis of hypertrophic cardiomyopathy was based on the demonstration of unexplained left, right or biventricular hypertrophy. An individual was diagnosed with sporadic disease if both parents were available for study and lacked criteria for diagnosis of hypertrophic cardiomyopathy.

Detection of mutations. The techniques used to screen the $\beta$ cardiac MHC gene coding sequence for mutations have been described in detail elsewhere $(20,21)$. The polymerase chain reaction (PCR) was used to amplify $\beta$ cardiac MHC gene or mRNA sequences from affected individuals' Epstein-Barr virus transformed lymphocytes. Both normal and mutationally altered $\beta$ cardiac MHC mRNA was detected by two rounds of PCR amplification. Amplified sequences were hybridized to RNA probes transcribed from a normal gene and RNase A protection assays performed as described previously $(20,21)$. The entire $\beta$ cardiac MHC coding sequence was examined in all probands, using both sense and antisense riboprobes.

Amplified DNAs that yielded abnormal RNase cleavage patterns were reanalyzed with new DNA isolates to exclude artifacts arising from PCR, and then subjected to dideoxy nucleotide sequence analysis. Where possible, the nucleotide change identified by sequencing was independently confirmed by demonstration of a predicted change in restriction enzyme site. Amino acid sequences were deduced from DNA sequences.

Genetic analysis of families. When a mutation was identified in a proband with sporadic hypertrophic cardiomyopathy its presence was sought in first-degree relatives by either RNase protection assays or restriction enzyme digestion. Paternity was confirmed by demonstration of inheritance of appropriate alleles at eight polymorphic dimeric short tandem repeat sequences (STRs): APOAII, APOCII, ASS, D1S103, D1S1 16, GCG, INT2, and PLA2 (23-29), using PCR conditions as described previously with end-labeled forward primers (23). Allele frequencies were calculated from a panel of 22 unrelated probands. The parental origin and subsequent inheritance of chromosomes bearing the de novo mutations in the probands was assessed by analysis of inheritance of alleles at polymorphisms within the $\beta$ cardiac MHC gene: dimeric STRs in the $5^{\prime}$ untranslated region (30) and in intron 24 (31) and the restriction fragment length polymorphism identified by probe pSC14 (32).

\section{Results}

Seven unrelated probands with sporadic hypertrophic cardiomyopathy were identified; all had typical features of hypertrophic cardiomyopathy with maximum left ventricular wall thickness ranging from 1.8 to $4.0 \mathrm{~cm}$ (median $3.0 \mathrm{~cm}$ ). The $\beta$ cardiac MHC gene was analyzed in each proband, using the RNase protection assay. $\beta$ cardiac MHC gene mutations were found in two of these probands, designated $H$ and AR.

\section{Clinical analyses}

Family $H$. Individual II-2 in family $\mathrm{H}$ was diagnosed with hypertrophic cardiomyopathy because of typical clinical features (Table I and Fig. 1). The proband's father (I-1) has a 10-year history of treatment for essential hypertension and symptomatic ischemic heart disease and underwent open heart surgery in 1974 for excision of a left atrial myxoma. A 12-lead electrocardiogram showed atrial flutter with minor repolarization changes; a two-dimensional echocardiogram demonstrated a dilated left atrium $(6 \mathrm{~cm})$ and a mildly dilated poorly contracting left ventricle without hypertrophy. Based on the absence of typical features of hypertrophic cardiomyopathy, and the presence of known cardiovascular disease, individual I-1 was diagnosed as unaffected by hypertrophic cardiomyopathy. Clinical analyses of the proband's mother (I-2) were entirely normal. Individuals I-1 and I-2 had not been studied previously. Previous analyses of the proband's children had identified one affected individual, III-2 (21). All other members of the family have an entirely normal clinical examination, EKG and echocardiogram.

Family $A R$. Individual II-1 in family AR also has typical clinical features of hypertrophic cardiomyopathy, with marked asymmetric septal hypertrophy and systolic anterior motion of the mitral valve (Table I). The proband's father, individual I-1, had a past medical history of myocardial infarction in 1979. A 12-lead electrocardiogram showed anteroseptal Q waves; there were no features of hypertrophic cardiomyopathy on two-dimensional echocardiogram. Clinical studies of all other first

Table I. Clinical Features of Affected Individuals and Their Parents in Families $H$ and $A R$

\begin{tabular}{|c|c|c|c|c|c|c|c|c|c|c|c|}
\hline \multirow[b]{2}{*}{ Subject } & \multirow[b]{2}{*}{ Age/Sex } & \multirow[b]{2}{*}{ Chest pain } & \multicolumn{2}{|c|}{ Clinical symptoms } & \multicolumn{3}{|c|}{ Electrocardiogram* } & \multicolumn{4}{|c|}{ 2-D echocardiogram ${ }^{\ddagger}$} \\
\hline & & & Dyspnea & Syncope & BBB & ABN Q & LVH $+/-\mathrm{ST}$ & MAX LVWT & RVH & SAM & LA SIZE \\
\hline & & & & & & & & $m m$ & & & $m m$ \\
\hline $\mathrm{H} \mathrm{I}-1^{8}$ & $73 \mathrm{M}$ & + & I & - & - & - & - & 14 & - & - & 60 \\
\hline H I-2 & $70 \mathrm{~F}$ & - & I & - & - & - & - & 9 & - & - & 32 \\
\hline H II-2 & $44 \mathrm{~F}$ & + & III & - & $\mathbf{L}$ & - & - & 18 & - & - & 50 \\
\hline H III-2 & $24 \mathrm{~F}$ & - & II & - & - & + & + & 28 & + & ++ & 42 \\
\hline AR I-1" & $70 \mathrm{M}$ & - & I & - & - & + & - & 11 & - & - & 30 \\
\hline AR I-2 & $63 \mathrm{~F}$ & - & I & - & - & - & - & 10 & - & - & 40 \\
\hline AR II-1 & $40 \mathrm{~F}$ & + & I & + & - & - & + & 38 & - & + & 38 \\
\hline
\end{tabular}

\footnotetext{
* Electrocardiographic abnormalities included bundle branch block (BBB; L, left), abnormal Q waves (ABN Q), and left ventricular hypertrophy with or without associated ST changes (LVH +/-ST).

‡ Echocardiographic findings included maximum left ventricular wall thickness (MAX LVWT), right ventricular hypertrophy (RVH), systolic anterior motion of the mitral valve (SAM; + incomplete, ++ complete), and left atrial size (LA SIZE).

${ }^{8}$ Electrocardiogram showed atrial flutter with repolarization changes (see text).

"Electrocardiogram showed anteroseptal Q waves due to previous myocardial infarction.
} 

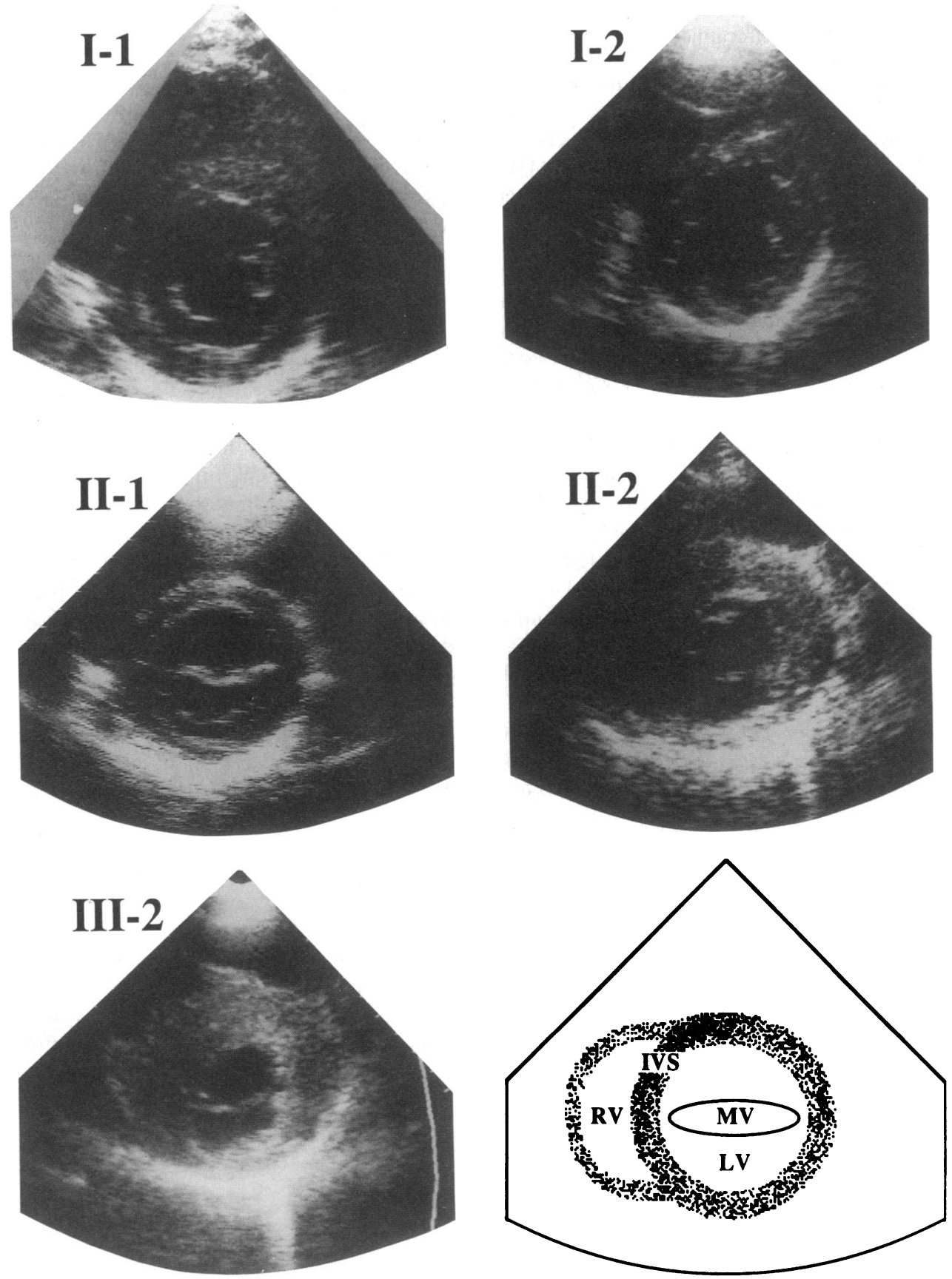

Figure 1. Echocardiograms of members of family $H$. Short-axis 2$D$ echocardiogram views taken at the level of the mitral valve leaflets are shown with a schematic illustrating the anatomical structures visualized (LV, left ventricle; MV, mitral valve; IVS, interventricular septum; RV, right ventricle). LV wall thickness is normal in individuals I-1, I-2, and II- 1 and abnormal in individuals II-2 and III-2. degree relatives, including the proband's mother and children, were entirely normal.

\section{Genetic analyses}

Family $H$. A $\beta$ cardiac myosin heavy chain gene mutation in the proband of family $\mathrm{H}$ was detected previously (21). This $\mathrm{G}$ to A transition in exon 23 (designated Glu924Lys) encodes a lysine residue rather than a glutamic acid residue at position 924 (numbered according to reference 30 ). The nucleotide substitution, as detected by an RNase protection assay of DNA amplified from exon 23 (Fig. 2), is present in both the proband (II-2) and her daughter (III-2), but neither parent. This result was confirmed by repeat analysis of independent samples from each family member. This mutation does not result in any change in restriction enzyme site.
Two possible explanations exist for the finding that neither parent bears the mutation present in the proband. Either the mutation has arisen de novo, or else has been inherited from a father other than individual I-1. To determine if individual I-1 was the biological father of individuals II-1 and II-2 we have characterized the alleles found in father (I-1), mother (I-2), son (II-1), and daughter (II-2) at eight polymorphic STRs spread throughout the genome (Methods). At each locus the children have inherited alleles present in the father's genome, rather than other alleles that exist in the general population (Fig. 3 and data not shown). From the frequency of the inherited alleles in the population we have calculated that the odds that a particular individual, chosen at random from the population, would have been able to produce the alleles found in individuals II- 1 or II- 2 are $<1$ in 100,000 . We conclude that 


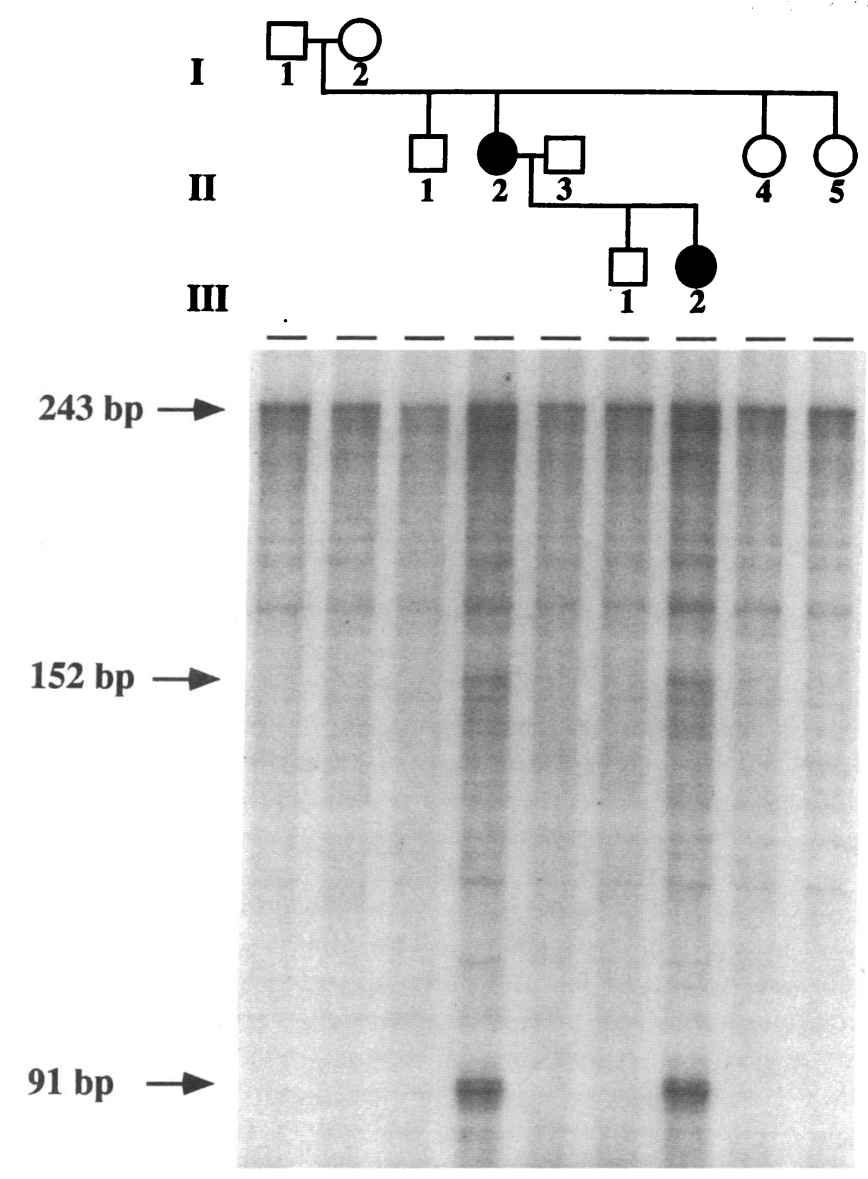

Figure 2. Detection of the Glu924Lys mutation in exon 23 in members of family $\mathrm{H}$ by $\mathrm{RNase}$ protection assay. The pedigree is shown above. Squares denote males and circles denote females; solid symbols denote those clinically affected and open symbols denote those unaffected. Below is an RNase protection assay of exon 23 DNA from each individual, in corresponding positions to those of the pedigree. Amplified DNA is hybridized to a ${ }^{32} \mathrm{P}$-labeled RNA probe of normal $\beta$ MHC sequence which is then digested with RNase A, resulting in cleavage at mismatched bases. The normal exon protects a 243 base fragment (arrow); the Glu924Lys mutation results in cleavage of this to fragments of 91 and 152 bases (arrows). Cleavage due to the nucleotide mismatch is present only in the clinically affected individuals II-2 and III-2.

individual I- 1 is the biological father of both the proband and her unaffected brother.

The parental origin of the chromosome bearing the de novo mutation was assessed by haplotype analysis of the $\beta$ cardiac MHC gene. Polymorphisms in a dimeric STR present in the 5 untranslated region (methods) demonstrate that the chromosome bearing the Glu924Lys mutation in the proband (II-2) was inherited from the father (I-1). This chromosome, which bears a normal copy of the $\beta$ cardiac MHC gene in the father, is also inherited in its normal state by an unaffected son (II-1) (data not shown). Thus this normal chromosome is associated with absence of hypertrophic cardiomyopathy in individuals I-1 and II-1, but once carrying the new Glu924Lys mutation is associated with the appearance of hypertrophic cardiomyopathy in individuals II-2 and III-2.

Family $A R$. Screening the $\beta$ cardiac MHC gene cDNA sequence in the proband of family AR (II-1) identified a novel abnormality on RNase protection. Nucleotide sequence analysis revealed this to be due to a $C$ to $T$ transition in exon 20 (affecting nucleotide residue 2253, numbered according to reference 33 ). This substitution encodes a change from the normal arginine amino acid residue to a cysteine in the mutant polypeptide, and is designated Arg723Cys. Arginine residue 723 is conserved among all known cardiac MHC's and all vertebrate striated muscle MHC's except the human perinatal and rabbit skeletal isoforms (34); mutation to a cysteine residue constitutes a nonconservative substitution with a change in net charge. This missense mutation, which is highly analogous to those others previously described, has not been seen in analyses of more than 180 normal chromosomes.

The nucleotide substitution involved in the Arg723Cys mutation is predicted to abolish a BcgI restriction enzyme site present in the normal cDNA sequence. Digestion of amplified cDNA sequences with this enzyme (Fig. 4) confirms the presence of the mutation in individual II-1 and also confirms that all other members of the family are genetically unaffected. The paternal relationship of individual I-1 to II-1 was confirmed with the eight polymorphic markers, providing odds of greater than 100,000 to 1 in favor of paternity. The $\beta$ cardiac MHC gene polymorphisms were not sufficiently informative in family AR to determine the parental origin of the chromosome that acquired the Arg723Cys mutation.

\section{Discussion}

These analyses identify two examples of missense mutations in the $\beta$ cardiac MHC genes of individuals with hypertrophic cardiomyopathy whose parents are clinically and genetically unaffected. We speculate that the $\beta$ cardiac MHC gene mutations in both probands arose in a germline cell as these mutations are expressed in cells of different lineages, including myocytes and peripheral leukocytes, as well as ovarian germ cells in the proband of family $\mathrm{H}$. Collectively, these data suggest that sporadic hypertrophic cardiomyopathy can result from new mutations that may then be inherited. In one family studied (AR), the affected proband is typical of cases of sporadic hypertrophic cardiomyopathy and no first-degree relatives are clinically or genetically affected. However because there is only a $50 \%$ chance that a given chromosome will be inherited by each child, the probability of a new mutation appearing in the subsequent generation is determined by the number of offspring. Documentation of germline mutations is frequently difficult in families of small size. Analyses of the $\mathrm{H}$ family demonstrated a de novo $\beta$ cardiac MHC gene mutation that is inherited; analyses of generations I and II identify sporadic disease while studies of generations II and III demonstrate a familial pattern. We conclude that de novo $\beta$ cardiac MHC gene mutations can cause sporadic hypertrophic cardiomyopathy; in at least some instances, these new mutations contribute to the incidence of familial hypertrophic cardiomyopathy.

The demonstration of de novo germline mutations in the $\beta$ cardiac MHC gene in individuals with sporadic disease is important in confirming that these mutations are directly responsible for producing hypertrophic cardiomyopathy. In families that coinherit hypertrophic cardiomyopathy and abnormalities of the MHC gene, the possibility exists that these abnormalities are merely linked to an unknown gene that is itself responsible for the phenotype. The evidence against this, although very 


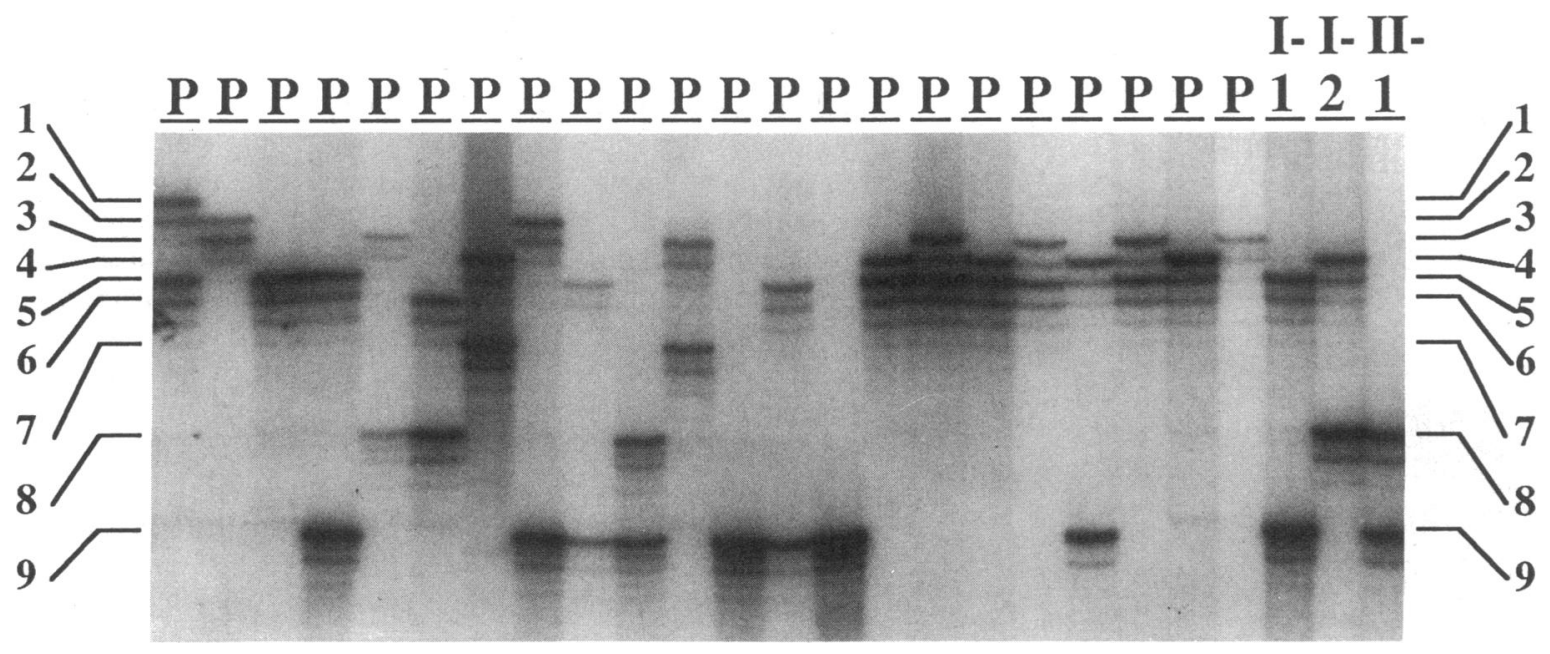

Figure 3. Example of STR sequence polymorphism used to confirm paternity. Alleles were typed by PCR amplification of a dimeric STR present in the APOC2 gene in 22 unrelated individuals and in family $\mathrm{H}$ members. The proband (II-1) inherits allele 8 from her mother (I-2) and therefore must inherit allele 9 from her father. This allele is present in individual I-1, and occurs in 10 of 44 alleles present in the unrelated probands ( $P$ ).

strong, is circumstantial: mutations have not been seen in unaffected individuals; mutations segregate concordant with disease status in large families; mutations affect conserved amino acid residues, frequently altering the charge (21). The evidence in families $\mathrm{H}$ and $\mathrm{AR}$ of a normal gene in unaffected individuals acquiring a de novo mutation in individuals who develop sporadic hypertrophic cardiomyopathy cannot be explained by such a chance association.

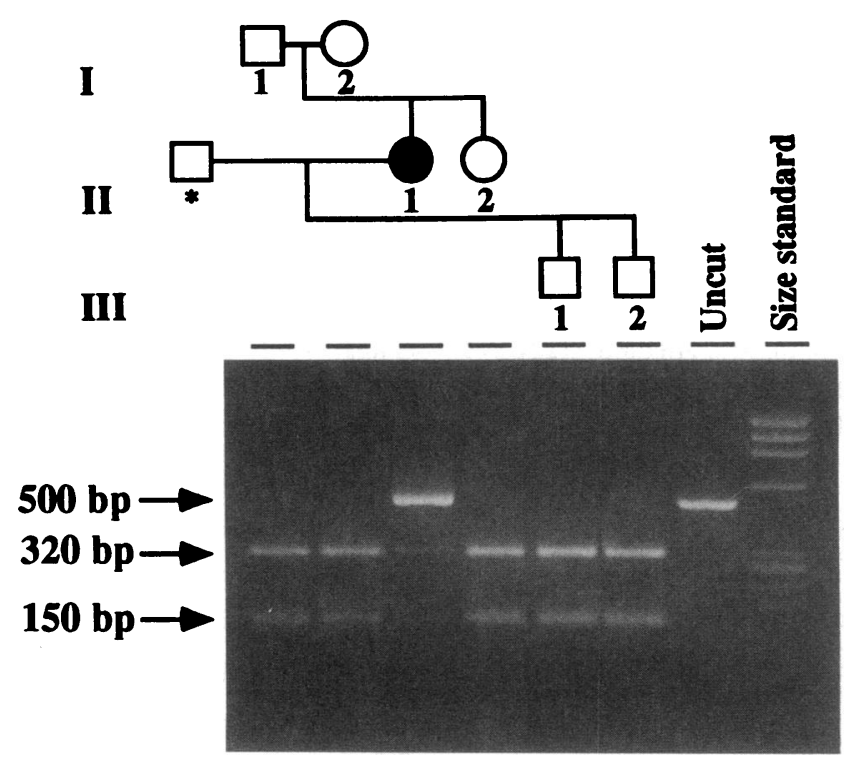

Figure 4. Detection of the Arg723Cys mutation in members of family AR by BcgI restriction enzyme digestion of PCR amplified cDNA. The pedigree is shown above, with symbols as for Fig. 2. A 500 basepair $\beta$ cardiac MHC cDNA is amplified as previously described, using primers numbered 1925 and 2424 (21). Digestion of a product of normal sequence generates fragments of 320 and 150 bp (arrows). The sample from individual II-1 contains these fragments but also the 500 base-pair fragment (arrow), because the Arg723Cys mutation abolishes the BcgI site on the affected chromosome. This mutation is not present in any other family member.
Because of the difficulties in clinically defining sporadic cases we are not able to determine the precise proportion of all sporadic hypertrophic cardiomyopathy that is caused by de novo mutations in the $\beta$ cardiac MHC gene. The finding of the two de novo mutations among the seven probands studied provides an estimate of the frequency of myosin mutations as a cause sporadic hypertrophic cardiomyopathy. It appears that mutations in other genes can cause sporadic hypertrophic cardiomyopathy with an identical phenotype. It seems likely that other loci, as yet unknown, that cause familial hypertrophic cardiomyopathy will be candidates for de novo mutations that cause sporadic hypertrophic cardiomyopathy.

Alternatively, sporadic cases may occur through etiologically distinct mechanisms. Somatic mutations in the $\beta$ cardiac MHC gene occurring during embryogenesis that affect the heart progenitor cells but not the germline might be a cause of sporadic disease. Given that such somatic mutations would be unlikely to be expressed in peripheral leukocytes, these would not be detected by the techniques used in this study. Undefined environmental factors might similarly cause a hypertrophic phenotype and contribute to the incidence of sporadic disease.

The finding of de novo mutations in the $\beta$ cardiac MHC gene in individuals with sporadic hypertrophic cardiomyopathy has clinical implications. A genetic diagnosis based on demonstration of a myosin heavy chain mutation should assist in difficult diagnoses. The problems of establishing the clinical diagnosis in individual $\mathrm{H} \mathrm{I}$-1 highlight this issue. Co-existing heart disease, especially hypertension, commonly confounds interpretation of clinical studies, and may prevent the diagnosis, or exclusion, of hypertrophic cardiomyopathy. The findings presented here suggest that the benefits of molecular genetic analysis of the $\beta$ cardiac MHC gene can be extended to a proportion of cases of sporadic hypertrophic cardiomyopathy. We have previously shown that particular mutations specifically influence survival in familial hypertrophic cardiomyopathy, with nonconservative amino acid substitutions being associated with a worse prognosis (21). In the absence of a family history to predict prognosis, the value of defining the diseasecausing mutation as a prognostic indicator will be especially 
great in sporadic hypertrophic cardiomyopathy. Moreover, if an individual was not clinically recognized as having sporadic disease the impression of a benign family history may have led to an inappropriate assumption of low risk status.

Genetic counseling for individuals with sporadic hypertrophic cardiomyopathy has not been previously feasible in the absence of an understanding of the etiology of the condition. On the assumption that sporadic disease was a separate, and perhaps nongenetic entity, the risk of transmission to offspring may have been considered low. Whereas individuals with sporadic hypertrophic cardiomyopathy caused by de novo germline mutations in the $\beta$ cardiac MHC gene will transmit the disease gene to $50 \%$ of offspring. Once a $\beta$ cardiac MHC mutation has been identified in a proband, with either sporadic or familial hypertrophic cardiomyopathy, the possibility exists of prenatal diagnosis or preclinical diagnosis in children.

We conclude that sporadic hypertrophic cardiomyopathy is within the spectrum of familial disease. De novo $\beta$ cardiac MHC gene mutations in germline cells contribute to the incidence of both the sporadic and familial variants of this disorder. Genetic analyses of sporadic cases of hypertrophic cardiomyopathy can help to confirm diagnoses and provide important genetic information to physicians and affected individuals.

\section{Acknowledgments}

These studies would not have been possible without the invaluable assistance of family members. We are grateful to Annie O'Donoghue and Shaughan Dickie for assembling data on family members and to Mohammad Miri and Tatjana Levi for technical assistance.

This work was supported by grants from the British Heart Foundation (Drs. Watkins and McKenna; Dr. Watkins is the recipient of a British Heart Foundation Clinical Scientist Fellowship), Deutsche Forschungsgemeinschaft (Dr. Thierfelder), National Institutes of Health (HL-46320 to Dr. J. G. Seidman; HL-41474 and HL-42467 to Dr. C. E. Seidman), Howard Hughes Medical Foundation (Dr. J. G. Seidman), the American Heart Association (Dr. C. E. Seidman) and Bristol Myers Squibb company.

\section{References}

1. Maron, B. J., and S. E. Epstein. 1979. Hypertrophic cardiomyopathy: a discussion of nomenclature. Am. J. Cardiol. 43:1242-1244.

2. Report of the WHO/ISFC task force on the definition and classification of cardiomyopathies. 1980. Br. Heart J. 44:672-673.

3. Ferrans, V. J., A. G. Morrow, and W. C. Roberts. 1972. Myocardial ultrastructure in idiopathic subaortic stenosis. A study of operatively excised left ventricular outflow tract muscle in 14 patients. Circulation. 45:769-792.

4. Davies, M. J. 1984. The current status of myocardial disarray in hypertrophic cardiomyopathy. Br. Heart J. 51:361-363.

5. Hollman, A., J. F. Goodwin, D. Teare, and J. W. Renwick. 1960. A family with obstructive cardiomyopathy (asymmetrical hypertrophy). Br. Heart J. 22:449-456.

6. Frank, S., and E. Braunwald. 1968. Idiopathic hypertrophic subaortic stenosis: clinical analysis of 126 patients with emphasis on natural history. Circulation. 37:759-788.

7. Henry, W. L., C. E. Clark, and S. E. Epstein. 1973. Asymmetric septal hypertrophy (ASH): echocardiographic identification of the pathognomonic anatomical abnormality of IHSS. Circulation. 47:225-233.

8. Maron, B. J., J. S. Gottdiener, and S. E. Epstein. 1981. Patterns and significance of distribution of left ventricular hypertrophy in hypertrophic cardiomyopathy: a wide angle, two dimensional echocardiographic study of 125 patients. Am. J. Cardiol. 48:418-428.

9. Shapiro, L., and W. J. McKenna. 1983. Distribution of left ventricular hypertrophy in hypertrophic cardiomyopathy: a two-dimensional echocardiographic study. J. Am. Coll. Cardiol. 2:437-444.

10. McKenna, W. J., A. Kleinebenne, P. Nihoyannopoulos, and R. Foale. 1988. Echocardiographic measurement of right ventricular wall thickness in hy- pertrophic cardiomyopathy: relation to clinical and prognostic features. J. Am. Coll. Cardiol. 11:351-358.

11. Pare, J. A. P., R. G. Fraser, W. J. Pirozynski, J. A. Shanks, and D. Stubington. 1961. Hereditary cardiovascular dysplasia: a form of familial cardiomyopathy. Am. J. Med. 31:37-62.

12. Horlick, L., N. J. Petrovich, and C. F. Bolton. 1966. Idiopathic hypertrophic subvalvular stenosis: a study of a family involving four generations: clinical, hemodynamic and pathologic observations. Am. J. Cardiol. 17:411-418.

13. Clark, C. E., W. L. Henry, and S. E. Epstein. 1973. Familial prevalence and genetic transmission of idiopathic hypertrophic subaortic stenosis. $N$. Engl. J. Med. 289:709-714.

14. Haughland, H., O.-J. Ohn, H. Boman, and E. Thorsby. 1986. Hypertrophic cardiomyopathy in three generations of a large Norwegian family: a clinical, echocardiographic and genetic study. Br. Heart J. 55:168-175.

15. Maron, B. J., A. J. Tajik, H. D. Ruttenberg, T. P. Graham, G. F. Attwood, B. E. Victorica, J. T. Lie, and W. C. Roberts. 1982. Hypertrophic cardiomyopathy in infants: clinical features and natural history. Circulation. 65:7-17.

16. Maron, B. J., P. F. Nichols, L. W. Pickle, Y. E. Wesley, and J. J. Mulvihill 1984. Patterns of inheritance in hypertrophic cardiomyopathy: assessment by M-mode and two-dimensional echocardiography. Am. J. Cardiol. 53:1087-1094.

17. Greaves, S. C., A. H. Roche, J. M. Neutze, R. M. Whitlock, and A. M. Veale. 1987. Inheritance of hypertrophic cardiomyopathy: a cross-sectional and M mode echocardiographic study of 50 families. Br. Heart J. 58:259-266.

18. Tanigawa, G., J. A. Jarcho, S. Kass, S. D. Solomon, H. P. Vosberg, J. G. Seidman, and C. E. Seidman. 1990. A molecular basis for familial hypertrophic cardiomyopathy: an alpha/beta cardiac myosin heavy chain hybrid gene. Cell. 62:991-998.

19. Geisterfer-Lowrance, A., S. Kass, G. Tanigawa, H. P. Vosberg, W. J. McKenna, J. G. Seidman, and C. E. Seidman. 1990. A molecular basis for familial hypertrophic cardiomyopathy: a beta cardiac myosin heavy chain gene missense mutation. Cell. 62:999-1006.

20. Rosenzweig, A., H. Watkins, D.-S. Hwang, M. Miri, W. J. McKenna, T. Traill, J. G. Seidman, and C. E. Seidman. 1991. Preclinical diagnosis of familial hypertrophic cardiomyopathy by genetic analysis of blood lymphocytes. $N$. Engl. J. Med. 325:1753-1760.

21. Watkins, H., A. Rosenzweig, D.-S. Hwang, T. Levi, W. J. McKenna, C. E. Seidman, and J. G. Seidman. 1992. Characteristics and prognostic significance of myosin missense mutations in familial hypertrophic cardiomyopathy. $N$. Engl. J. Med. 326:1108-1114.

22. Jarcho, J. A., W. J. McKenna, J. A. P. Pare, S. D. Solomon, R. F. Holcombe, S. Dickie, T. Levi, H. Donis-Keller, J. G. Seidman, and C. E. Seidman. 1989. Mapping a gene for familial hypertrophic cardiomyopathy to chromosome 14q1. N. Engl. J. Med. 321:1372-1378.

23. Weber, J. L., and P. E. May. 1989. Abundant class of human DNA polymorphisms which can be typed using the polymerase chain reaction. Am. J. Hum. Genet. 44:388-396.

24. Yuille, M. A. R., R. M. Hampson, R. M. Harris, N. A. Affara, J. R. W. Yates, and M. A. Ferguson-Smith. 1990. CA repeat polymorphism at the ASS locus. Nucleic Acids Res. 18:7472.

25. Weber, J. L., A. E. Kwitek, and P. E. May. 1990. Dinucleotide repeat polymorphism at the D1S103 locus. Nucleic Acids Res. 18:2199.

26. Sharma, V., L. Allen, R. E. Magenis, and M. Litt. 1991. A dinucleotide repeat polymorphism at the DIS116 locus. Nucleic Acids Res. 19:1169.

27. Polymeropoulos, M. H., D. S. Rath, H. Xiao, and C. R. Merrill. 1991 Dinucleotide repeat polymorphism at the human preproglucagon gene. Nucleic Acids Res. 19:688.

28. Polymeropoulos, M. H., H. Xiao, D. S. Rath, and C. R. Merrill. 1990 Dinucleotide repeat polymorphism at the int-2 proto-oncogene locus (INT-2). Nucleic Acids Res. 18:7468.

29. Polymeropoulos, M. H., D. S. Rath, H. Xiao, and C. R. Merrill. 1990 Trinucleotide repeat polymorphism at the human pancreatic phospholipase A-2 gene (PLA2). Nucleic Acids Res. 18:7468.

30. Polymeropoulos, M. H., H. Xiao, D. S. Rath, and C. R. Merrill. 1991. Dinucleotide repeat polymorphism at the human cardiac beta-myosin gene. $\mathrm{Nu}$ cleic Acids Res. 19:4019.

31. Epstein, N. D., L. Fananapazir, H. J. Lin, J. Mulvihill, R. White, J.-M Lalouel, R. P. Lifton, A. W. Nienhuis, and M. Leppert. 1992. Evidence of genetic heterogeneity in five kindreds with familial hypertrophic cardiomyopathy. Circulation. 85:635-647.

32. Solomon, S. D., A. A. T. Geisterfer-Lowrance, H.-P. Vosberg, G. Hiller, J. A. Jarcho, C. C. Morton, W. O. McBride, A. L. Mitchell, A. E. Bale, W. J. McKenna, et al. 1990. A locus for familial hypertrophic cardiomyopathy is closely linked to the cardiac myosin heavy chain genes, CRI-L436, and CRI-L329 on chromosome 14 at q11-q12. Am. J. Hum. Genet. 47:389-394.

33. Jaenicke, T., K. W. Diederich, W. Haas, J. Schleich, P. Lichter, and H.-P. Vosberg. 1990. The complete sequence of the human $\beta$ myosin heavy chain gene and an analysis of its product. Genomics. 8:194-206.

34. Devereux, J., P. Haeberli, and O. Smithies. 1984. A comprehensive set of sequence analysis programs for the VAX. Nucleic Acids Res. 12:387-395. 